Check for updates

Cite this: Mater. Chem. Front. 2019, 3, 2010

Received 28th June 2019, Accepted 24th August 2019

DOI: $10.1039 / c 9 q m 00419 j$

rsc.li/frontiers-materials

\section{Selenium-containing supra-amphiphiles}

\author{
Wei Cao (D)*b and Huaping Xu (D)*a
}

Supra-amphiphiles formed by noncovalent interactions are a versatile bottom-up fabrication strategy for functional soft materials. Selenium chemistry possesses many unique properties and selenium-containing polymers made by covalent synthesis have developed enormously in the past few decades. Supra-amphiphiles built on selenium-containing species offer more opportunities for functional systems without too many synthetic efforts. This review will give a brief overview of different types of selenium-containing supraamphiphiles based on various driving forces. We will discuss structure-property-function relationships. The key question we want to address is how supra-amphiphile and selenium chemistry could impact research areas including controlled delivery, dynamic materials, tunable surfaces, nanomedicine, etc.

\section{Introduction}

Amphiphiles are molecules used extensively in technology and everyday life in which the hydrophilic and hydrophobic parts are connected by covalent bonds. ${ }^{1}$ In supra-amphiphiles, the building blocks could be connected through noncovalent linkages, such as electrostatic and van der Waals interactions, hydrogen bonds, coordination bonds, charge transfer interactions, host-guest

\footnotetext{
${ }^{a}$ Key Lab of Organic Optoelectronics and Molecular Engineering, Department of Chemistry, Tsinghua University, Beijing, 100084, China. E-mail: xuhuaping@mail.tsinghua.edu.cn

${ }^{b}$ Department of Chemistry, Northwestern University, Evanston, Illinois 60208, USA. E-mail:wei.cao@northwestern.edu
}

interactions, etc., to create amphiphiles that can self-assemble into functional systems. ${ }^{2-4}$ Self-assembly is a thermodynamically driven self-organization process of amphiphiles to make an ordered structure and complex functions with minimal energy input, ${ }^{5,6}$ while the supra-amphiphiles can be viewed as a hierarchical self-assembly process. ${ }^{7}$ The noncovalent nature simplifies the tedious synthetic procedure and allows easy incorporation of different functions into an integrated system. ${ }^{8,9}$

The past decade has witnessed the flourishing of seleniumcontaining polymers. By making block copolymers with selenium located in the backbone, chemists developed a series of seleniumcontaining polymers with different topologies. ${ }^{10,11}$ Their multiresponsiveness under mild conditions makes selenium-containing

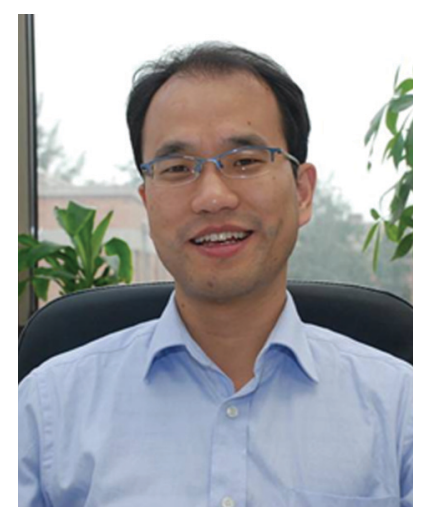

Huaping Xu
Prof. Huaping $X u$ received his $B S$ Wei Cao obtained his BS degree from Jilin University, China, in 2011. In 2016, he completed his PhD at the Department of Chemistry, Tsinghua University, under the supervision of Prof. Huaping $\mathrm{Xu}$. His thesis work involved oxidation/coordination responsive selenium/tellurium containing polymer self-assemblies. After a short stint at ULCA in Prof. Ellen Sletten's group working on flourous dyes, he moved to Northwestern University in 2018 as a postdoc scholar at Prof. Nathan Gianneschi's lab. His research interests are self-assembled functional nanomaterials for bioapplications. in 2001 and PhD degree in 2006 from Jilin University, under the supervision of Prof. Xi Zhang. In 2006, he joined Prof. David N. Reinhoudt and Prof. Jurriaan Huskens's group at the University of Twente as a postdoc. Since 2008, he has worked at Tsinghua University. In 2014, he received the Natural Science Fund for Outstanding Young Scholars from NSFC. In 2017, he was enrolled in Leading Talent of National Highlevel personnel of special support program ("people plan"). He is an Associate Editor of ACS Biomaterials Science \& Engineering. His current research is focused on selenium/tellurium-containing polymers. 
polymers emerging biomaterials for various applications, such as in redox-responsive drug delivery vehicles, ${ }^{12-15}$ highly efficient enzyme mimics, ${ }^{16,17}$ dynamic self-healing elastomers, ${ }^{18}$ triggered degradable polymeric vehicles, ${ }^{19,20}$ etc. As an alternative to the covalent selenium amphiphiles, developing noncovalent supra-amphiphiles is also an attractive approach to incorporate the rich chemical and biological functions of selenium chemistry into complex systems.

Selenium-containing species have many unique chemical features. Although selenium and sulfur share some chemical properties in terms of ionic radius and multiple available oxidation states, their differences are striking. One important difference is that the Pauling electronegativity of selenium (2.55) is lower than that of sulfur (Pauling electronegativity of $\mathrm{S}$ is 2.58), ${ }^{21}$ making the selenium compounds in low oxidation states more sensitive to oxidation. Also, selenium related chemical bonds have lower bond dissociation energy compared with the sulfur or carbon analogues. ${ }^{22}$ Selenium has a polarizability volume of 3.8 Angstrom, and is, thus, softer than sulfur (2.9 Angstrom). ${ }^{23}$ In addition, selenium is a better nucleophile and electrophile than sulfur. $^{24}$

Apart from intrinsic chemical properties, selenium species also have important biological functions. In nature, the human proteome codes for up to 25 proteins contain selenocysteine. ${ }^{25}$ A family of selenium-containing enzymes, glutathione peroxidase (GPx), protects organisms from oxidative stress. GPx was found to rely on selenocysteine as the active site for catalyzing the decomposition of peroxides. ${ }^{26}$ Other natural proteins include mammalian thioredoxin reductase, formate dehydrogenase of E. coli, etc. ${ }^{25}$ In addition, selenium chemistry is also powerful in synthetic proteins. Selenocysteine has a lower $\mathrm{p} K_{\mathrm{a}}(5.3)$ than cysteine $\left(\mathrm{p} K_{\mathrm{a}}=8.3\right)$, which makes selenocysteine more nucleophilic under neutral and even acidic conditions, and further facilitates selenocysteine to be incorporated into semisynthetic protein using native chemical ligations. $^{27}$

Designing supra-amphiphiles containing selenium species could incorporate the rich chemical properties and biofunctions into multi-level self-assembled systems. The supra-amphiphile strategy could broaden the application of selenium chemistry without sophisticated chemical synthetic efforts. Herein, we review the design, synthesis, and applications of a new class of multi-functional selenium-containing supra-amphiphiles built on non-covalent interactions. They can either be made based on conventional non-covalent interactions, such as hydrogen bonding, electrostatic interactions, or novel Se $\cdots \mathrm{X}$ interactions (Scheme 1). The review illustrates the design principles and noncovalent fabrication of the selenium-containing supraamphiphiles, highlighting the facile fabrication and rich functions due to the dynamic selenium chemistry and noncovalent nature of supra-amphiphiles. The present review is an attempt to help understand the structure-property-function relationships of these selenium-containing supra-amphiphiles from the bottom up. The key goal is to elucidate how the selenium-containing supraamphiphiles could improve the dynamic properties and response to external stimuli. We hope this line of research will shed light on some important fundamental and application challenges we meet in biomaterials and synthetic materials science.

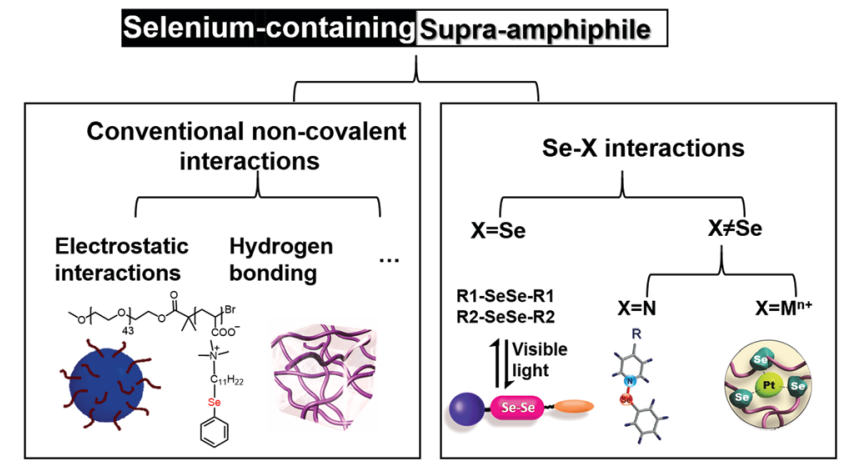

Scheme 1 Selenium-containing supra-amphiphiles based on different noncovalent interactions.

\section{Using conventional noncovalent interactions to incorporate selenium-containing species into supra-amphiphiles}

Since the first report of a diselenide containing block copolymer in $2010,{ }^{12}$ the unique redox response of selenium-containing polymers has attracted more and more attention from polymer chemists. $\mathrm{Xu}$ and Zhang reported a side chain selenium-containing polymeric supra-amphiphile (Fig. 1). ${ }^{28}$ Based on electrostatic interactions of the poly(ethylene glycol)- $b$-acrylic acid (PEG- $b$-PAA) and a selenium-containing surfactant, a polymeric supra-amphiphile was fabricated and could self-assemble into stable micelles in water as visualized by a Cryo-TEM (transmission electron microscope). Upon adding $0.1 \% \quad \mathrm{H}_{2} \mathrm{O}_{2}$, the seleniumcontaining small molecules were oxidized to selenoxide, and therefore became more hydrophilic. The amphiphilicity change leads to disassembly of the micelles and the subsequent release of loaded fluorescein. In this way, the oxidation response of selenium-containing species could be incorporated into supraamphiphiles easily.

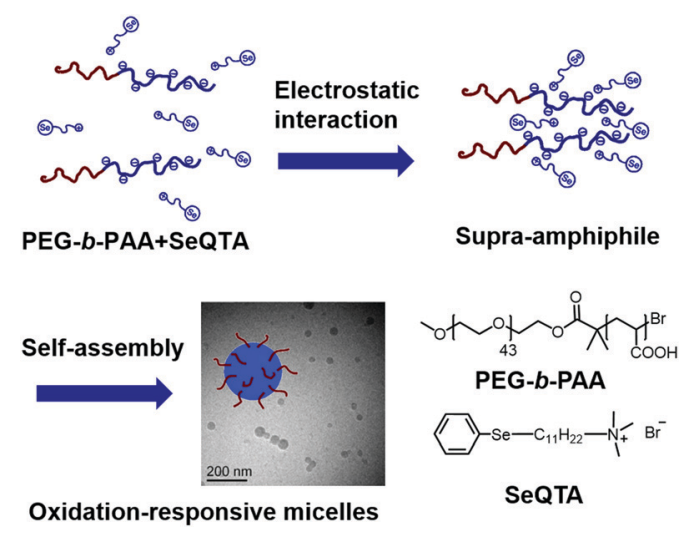

Fig. 1 Oxidation responsive supra-amphiphiles based on the electrostatic interactions between a double hydrophilic polymer and a seleniumcontaining amphiphile. The supra-amphiphile could self-assemble into spherical micelles. Adapted with permission from ref. 28. Copyright 2010 American Chemical Society. 


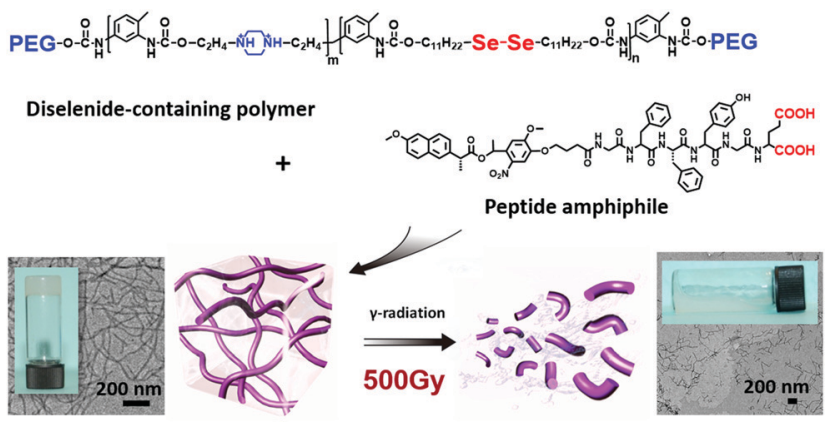

Fig. 2 Gamma-ray responsive hydrogel of supra-amphiphiles formed by a diselenide-containing polymer and a peptide amphiphile. Adapted with permission from ref. 29. Copyright 2013 Wiley.

Apart from the oxidation response, selenium-containing polymers also exhibit a gamma-ray response. Gamma ray is a therapeutic radiation in clinical anticancer treatment due to its high frequency and high penetration depth in tissues. Developing some materials that can change morphology under gamma radiation could potentially be used for radiation-induced drug delivery and therefore work as a platform for combined chemoand radio-therapies. $\mathrm{Xu}$, Yang, and coworkers reported a supramolecular hydrogel that exhibited a $\gamma$-radiation-induced gel-sol transition (Fig. 2). ${ }^{29}$ A peptide amphiphile with negative charges was designed and synthesized. After complexing with positively charged diselenide containing polymers through electrostatic interactions and $\mathrm{H}$ bonding interactions, the supramolecular complex reached the required amphiphilicity balance of a gelator. Cryo-TEM showed the fibrous nature of the hydrogel. Radiation of 500 Gy $\gamma$-ray resulted in the transition to the sol state accompanied by the breaking down of long nanofibers. Mechanistic studies revealed that $\gamma$-ray generated reactive oxygen species in water and caused the cleavage of the diselenide bond on the polymer backbone, further resulting in the degradation of the supramolecular network. The diselenide bonds are essential for the $\gamma$-ray response as the disulfide control gel does not exhibit the same responsiveness. The supra-amphiphile with diselenide bonds on the polymer backbones could provide another approach to broaden the selenium-containing polymer materials for biomedicine related applications. Further utilizing the more sensitive tellurium containing polymers could enable a self-assembly morphological change $^{30}$ and triggered drug release ${ }^{31}$ at clinically relevant gamma ray dosage (2 Gy).

\section{Using Se...X dynamic interactions to fabricate supra-amphiphiles}

It's of high importance to develop supra-amphiphiles based on new dynamic/non-covalent interactions. The new driving force could facilitate the development of a lot more exotic supraamphiphiles. Selenium is a ligand for coordination chemistry due to the vacant $4 \mathrm{~d}$ orbitals. And selenium-containing bonds have low bond dissociation energies, making them attractive as dynamic covalent bonds. A series of Se-X bonds were used to develop supra-amphiphiles, in which the X could be Se or other elements, including nitrogen, metal cations, etc.

\section{When $\mathrm{X}=$ Se}

Since selenium resembles sulfur in its chemical properties, we hypothesized that diselenide bonds would behave similarly to disulfide bonds and serve as dynamic covalent bonds. $\mathrm{Xu}$ et al. revealed the dynamic exchange nature of the diselenide bond under very mild visible light. ${ }^{32,33}$ Two moles of symmetric diselenide-containing compounds, R1SeSeR1 and R2SeSeR2, can undergo a metathesis reaction, yielding 1 mole of the asymmetric product (R1SeSeR2) under visible light without any additional catalysts (Fig. 3). The final composition of the reaction was determined by NMR spectra to be 1:1:2 for R1SeSeR1, R2SeSeR2, and the asymmetric R1SeSeR2. The reaction proceeds through a radical mechanism. A detailed discussion on this topic is beyond the scope of this review, and readers are referred to the recent literature. ${ }^{33-35}$ The diselenide chemistry is a new candidate for dynamic covalent materials.

The novel chemistry makes it possible to synthesize amphiphilic block copolymers by simply mixing PS-SeSe-PS and PEG-SeSe-PEG (Fig. 3B) together. Although it is still a mixture after the exchange reaction, the block copolymer PS-SeSe-PEG could self-assemble into vesicles in water. The polymeric supraamphiphile was held together by the weak Se-Se bond, which could be disrupted by the mechanical force as a result of osmotic pressure induced by strong electrolytes, like $\mathrm{NaCl}$, $\mathrm{NaBr}$ and $\mathrm{CaCl}_{2} \cdot{ }^{36}$ In contrast, the control polymer connected by an ester bond remains intact under the same conditions. This example demonstrated that the diselenide bonds could be used as a mechanophore and that the supra-amphiphiles built on diselenide bonds could have various responses towards different stimuli based on their dynamic covalent nature.

Yet using diselenide bonds to make asymmetric amphiphiles proved to be a challenge due to the constant exchange reaction triggered by visible light. Visible light is the most ubiquitous electromagnetic radiation in our daily environment.

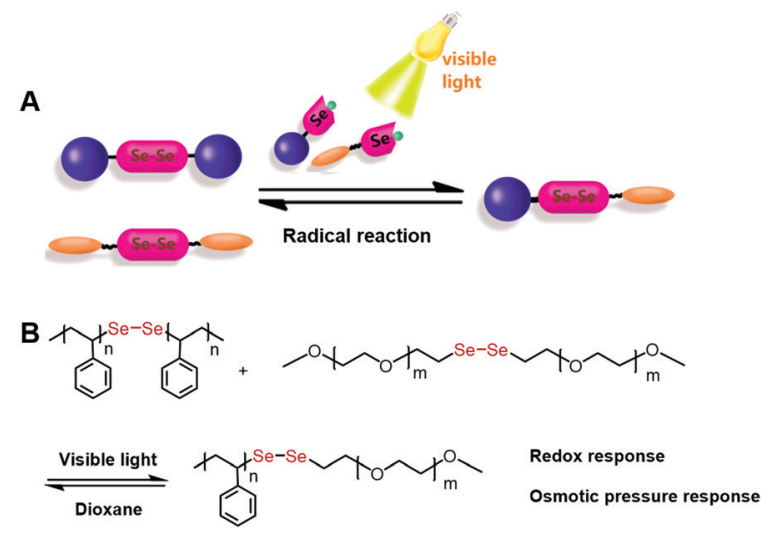

Fig. 3 (A) Schematic illustration of the dynamic exchange reaction between different diselenide molecules under visible light. Reprinted with permission from ref. 32. Copyright 2014 Wiley. (B) Using the diselenide exchange reaction to make amphiphilic block copolymers. 
A
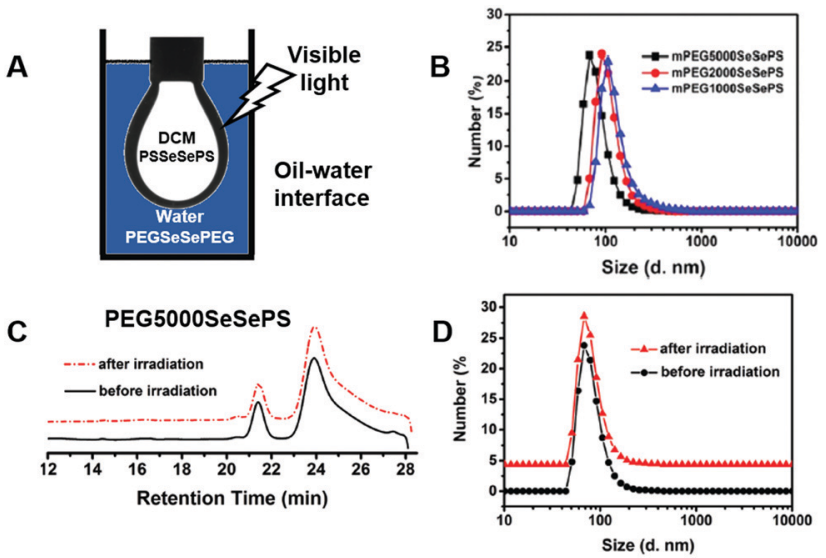

E

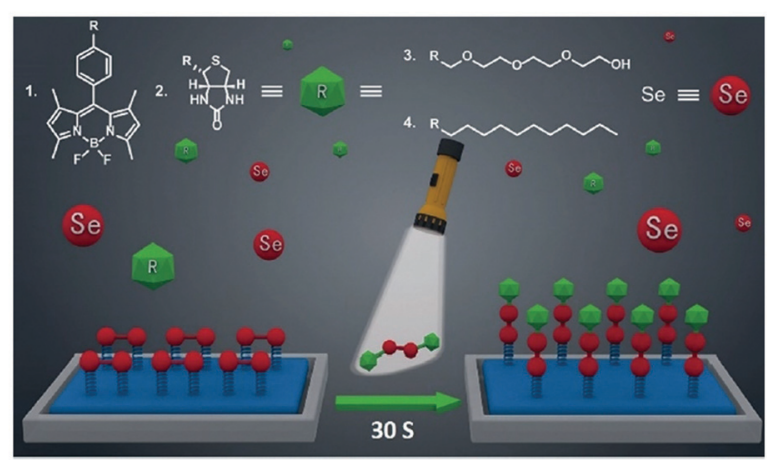

Fig. 4 Diselenide covalent chemistry at the interface. (A) Stabilizing an asymmetric diselenide-containing polymer by micelle formation. Reprinted with permission from ref. 38. Copyright 2016 Royal Society of Chemistry (Great Britain). (B) DLS of different micelles. (C) GPC and (D) DLS plots for stability test of the PEG5000SeSePS micelles after irradiation. (E) Using diselenide exchange for fast surface modification under visible light. Reprinted with permission from ref. 39. Copyright 2018 Wiley.

In addition, the activation energy of the diselenide exchange reaction should be very low. ${ }^{37}$ To push the equilibrium of the diselenide exchange reaction under mild conditions is certainly a big step forward for this new metathesis chemistry.

$\mathrm{Xu}$ et al. reported using an oil/water or air/solid interface to stabilize the asymmetric supra-amphiphiles connected by the diselenide bond (Fig. 4). ${ }^{38}$ The diselenide bond exchange reaction on the oil/water interface was confirmed by a pendant drop test. The dichloromethane drop contained water-insoluble benzyl diselenide, while the water phase is a tetra ethylene glycol diselenide solution. The surface tension decreased over time under visible light, indicating the formation of the amphiphilic surfactant on the interface. Moving forward to using mono- or diselenide containing PEG-SeSe-PEG and PS-SeSe-PS, the amphiphilic PEG-Se-Se-PS could also be made on the interface and remained stable even after photo-radiation according to the GPC and DLS results. The oil-water interface stabilized supraamphiphile still undergoes a sensitive redox response, providing a useful tool to expand the methodology for fabricating supraamphiphiles. The diselenide containing supra-amphiphiles could be applied to solid substrate surface modification to change the surface wetting properties (Fig. 4E). Under 30 second visible light illumination, liquid motion control was achieved by altering the surface wettability with diselenide metathesis reactions. ${ }^{39}$ The same strategy could be used for surface bioconjugation to immobilize biotin and streptavidin on ITO conductive film glass for further sensing and diagnosing.

\section{When $X \neq$ Se}

Asymmetric Se $\cdots \mathrm{X}$ interactions are also studied along with their application in supra-amphiphiles and polymer systems. Here $\mathrm{X}$ could be nitrogen, metal ions and so on.

\section{$\mathbf{X}=\mathbf{N}$}

Intra-molecular Se $\cdots \mathrm{N}$ interactions are important for GPx and its mimics, ${ }^{40,41}$ yet inter-molecular Se $\cdots \mathrm{N}$ interactions are far less studied. Xu and Zhang reported using Se $\cdots \mathrm{N}$ noncovalent interactions to fabricate well-defined 2-dimensional crystalline nanosheets (Fig. 5). ${ }^{42}$ When a macrocycle azacalix[6]pyridine (APy6) and a three-armed Se containing amphiphile (EG-Se) were mixed together, unexpected 2D nanosheets were observed in water. AFM (atomic force microscopy) and TEM result showed that the sheets were 1-2 $\mu \mathrm{m}$ long and $300-500 \mathrm{~nm}$ wide. The TEM based electron diffraction pattern suggested that they have a crystal structure. Powder X-ray diffraction (XRD) results indicated that the larger distance is the same as in single crystals of APy6. X-ray photoelectron spectroscopy (XPS) and Fourier transform infrared (FT-IR) spectroscopy could further confirm the Se $\cdots \mathrm{N}$ non-covalent interactions between the two species. The ESI-Q-TOF spectrum showed the 1:1 complex of EG-Se and APy6. The EG-Se/APy6 supramolecular complex, although not the dominant species in the self-assembly structures, adsorbed onto the surface of the nanocrystal sheets and prevented the growth of the face packing dimension. Control experiments of substituting the selenium with oxygen or protonating the nitrogen on the APy6 failed to yield the nanosheets, suggesting
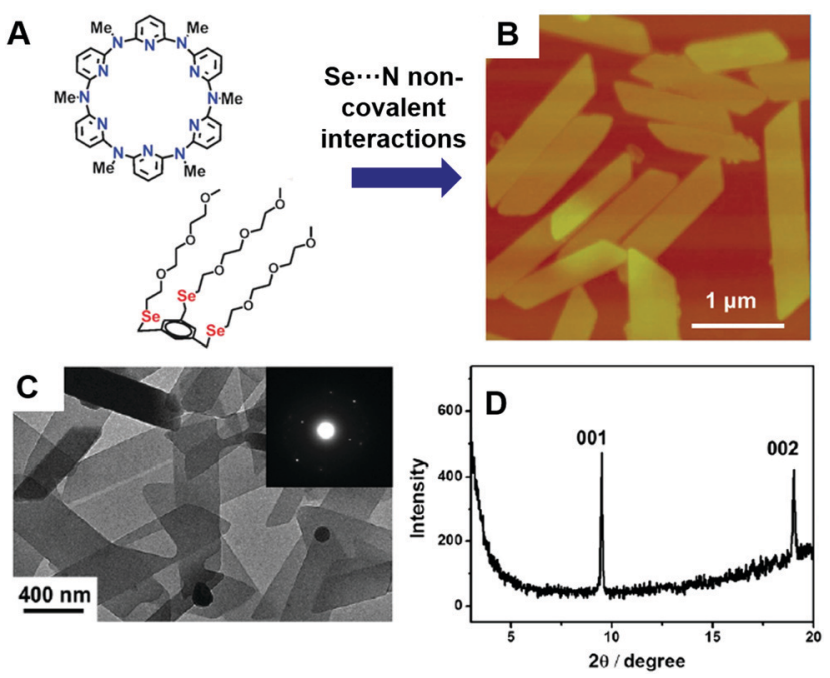

Fig. 5 (A) Se $\cdots \mathrm{N}$ non-covalent interactions assisted the fabrication of well-defined crystalline azacalixarene nanosheets. (B) AFM image of the nanosheets. (C) TEM electron diffraction pattern and (D) XRD of the sheets indicate the crystalline structure. Reprinted with permission from ref. 42. Copyright 2012 Royal Society of Chemistry (Great Britain). 

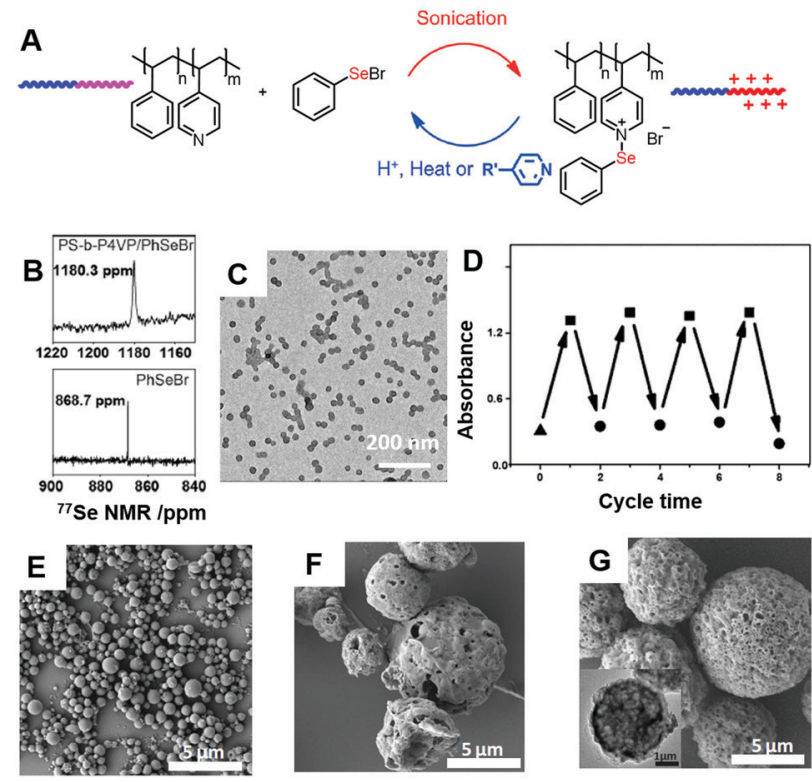

Fig. 6 Supra-amphiphiles formed based on Se-N dynamic covalent bonds (A) and the application in tuning the emulsion properties. (B) ${ }^{77} \mathrm{Se}$ NMR spectra of PhSeBr and the supra-amphiphiles formed by PhSeBr and PS- $b-$ P4VP. (C) The TEM image of the micelles formed in dichloromethane. (D) Dynamic nature of the Se-N bonds. $(E-G)$ are the SEM image of emulsions made by the oil phase containing different amphiphilic blends with the pyridine group to $\mathrm{PhSeBr}$ molar ratios from $1: 0.2,1: 0.5$ to $1: 1$, respectively. Reprinted with permission from ref. 44. Copyright 2014 Wiley.

that the Se $\cdots \mathrm{N}$ supra-amphiphiles played an important role in the 2D material synthesis.

In contrast to the non-covalent Se $\cdots \mathrm{N}$ interactions, a Se-N dynamic covalent bond is quickly formed when a phenylselenyl halogen species reacts with pyridine. ${ }^{43} \mathrm{PhSeX}(\mathrm{X}$ is $\mathrm{Br}$ or $\mathrm{Cl})$ could react with polystyrene- $b$-poly(4-vinylpyridine) (PS- $b$-P4VP) in dichloromethane (Fig. 6). The significant shift in ${ }^{77}$ Se NMR after the reaction suggested the covalent nature of the new bond. The Se-N bond increased the polarity of the P4VP block, rendering the self-assembled structures in dichloromethane as shown in the TEM image. The supra-amphiphile could be broken if heat is applied as indicated by temperature dependent FT-IR. Also, the dynamic covalent Se-N bond could reversibly break and reform under a Brønsted acid or base. As shown by UV-vis absorbance at $470 \mathrm{~nm}$, the dynamic process could last over at least 4 cycles.

The Se-N dynamic covalent bond is a useful chemical tool for amphiphilicity tuning towards emulsion property control. Using the PhSeBr/PS-P4VP supra-amphiphile as a facilely tunable surfactant, Xu and Wang successfully obtained a series of emulsions with different sizes and core structures (Fig. 6E-G). ${ }^{44}$ Porous microparticles with a high surface area can be obtained in one step. Changing the ratio of the Se-N bond formation not only provides a strong handle in tuning the amphiphilicity, but also changes the catalytic activity for peroxide decomposition. Particles with $1: 1$ ratio of $\mathrm{PhSeBr}$ and the pyridine moiety showed the highest catalytic activity and had a better performance with hydrophobic substances due to the w/o/w complex structure. The example demonstrates the advantage of utilizing a supra-amphiphile as an adjustable surfactant to tune the material nanostructure and to achieve complex functions simultaneously.

$\mathbf{X}=\mathbf{M}^{n+}$

As a chalcogen element, selenium can bind with multiple metal ions. According to the hard and soft acids and bases (HSAB) theory, selenium-containing compounds are soft Lewis base. Therefore, selenium should coordinate better with more polarizable transition metal cations. The coordination is very important considering that there are many metal drugs in clinical use.

$\mathrm{Xu}$, Sun, et al. reported a coordination responsive drug delivery system based on the coordination of monoselenide containing polymers and platinum cations (Fig. 7) ${ }^{45}$ The coordination interaction was confirmed by ${ }^{1} \mathrm{H}$ NMR, ${ }^{77}$ Se NMR, and MALDI-TOF spectra. The coordination bond increased the stability of micelles against high ionic strength. Platinum coordination also increased the hydrophilicity of the monoselenide containing polymers, as indicated by the change in critical aggregation concentration. When loaded with doxorubicin, the release of $\mathrm{Pt}^{2+}$ and doxorubicin could be triggered simultaneously when other competitive ligands are present. The supra-amphiphile could work as a coordination responsive drug delivery platform for multidrug therapy. Other competitive ligands or metal drugs might also work for this delivery system. $^{46}$

Later a coordination supra-amphiphile of a three-armed selenide small molecule compound, EG-Se, with cisplatin was reported as a highly selective anticancer drug. ${ }^{47}$ Compared with polymers, small molecules have defined composition and have minimal batch to batch variations, making them more promising for FDA approval. The coordination structure of EG-Se and cisplatin was studied and is shown in Fig. 8. The coordination complex was found to kill $96 \%$ of human liver carcinoma cells (HepG2) in an in vitro experiment, while for normal liver cells (L-02), only $21 \%$ were killed. The mechanistic study indicated that the supra-amphiphile could induce high reactive oxygen species (ROS) expression in HepG2 cells, further causing apoptosis. Studies on the mitochondria membrane

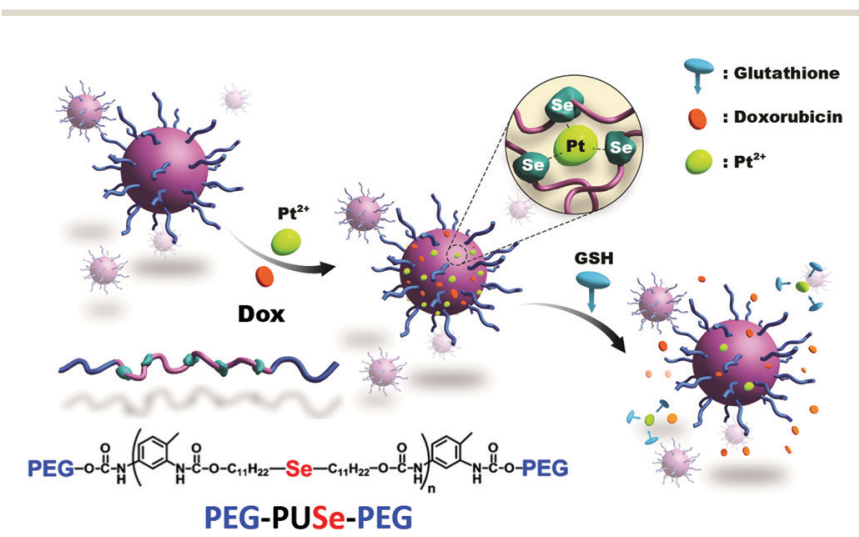

Fig. 7 Coordination-responsive micelles for controlled drug release built on the coordination interactions between selenium-containing polymers and platinum cations. Reprinted with permission from ref. 45. Copyright 2012 Royal Society of Chemistry (Great Britain). 
A

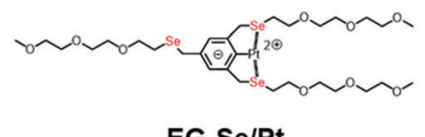

EG-Se/Pt

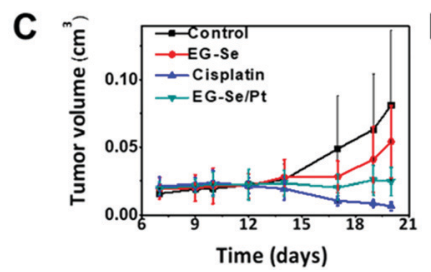

B

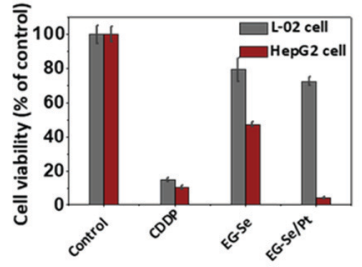

D

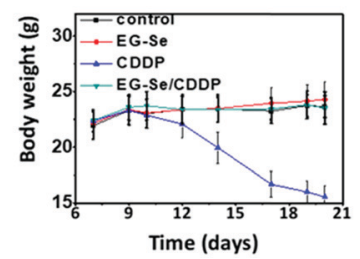

Fig. 8 A selenium-platinum coordination compound (A) as a novel selective anticancer drug. (B) The drug EG-Se/Pt could selectively kill cancer cells. In vitro experiment demonstrated high efficacy (C) and low toxicity (D) for mouse models. Reprinted with permission from ref. 47. Copyright 2014 Wiley.

potential revealed that the drug could depolarize the mitochondria membrane in HepG2 cells, while in L-02 cells, the effect is minimal. ${ }^{48}$ Because of the supramolecular nature of the complex, we can easily switch platinum with other metal ions. Yet neither copper(II) or nickel(II) complex showed a similar selectivity. The EG-Se/Pt complex has broad-spectrum anticancer activity in breast cancer cells (MCF-7), human lung adenocarcinoma cells (A549) and even colon carcinoma cell groups (Ht-29), which is resistant to cisplatin. In vivo studies demonstrated that the EG-Se/Pt complex showed high efficacy at inhibiting tumor growth in HepG2 tumor-bearing nude mice. Low toxicity was observed in healthy BALB/c mice with normal immunity. The supramolecular drug could provide a selective therapeutic strategy against cancer. Further mechanistic studies on the uptake, DNA intercalation, etc. will provide more fundamental insight for the rational design of selective anticancer therapeutics.

Cisplatin could coordinate with the poly-lysine dendrimers with monoselenide located in the core (Fig. 9). ${ }^{49}$ Dendrimers

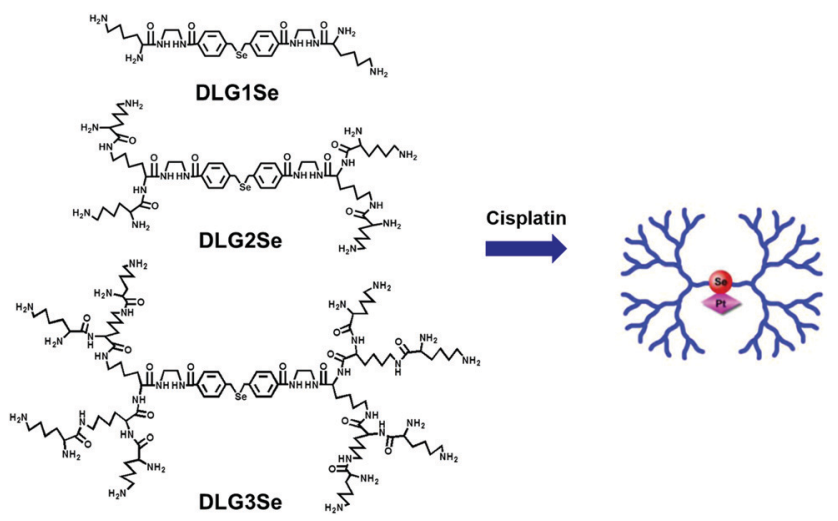

Fig. 9 Selenium-platinum coordination dendrimers with controlled anticancer activity. Reprinted with permission from ref. 49. Copyright 2016 American Chemical Society. are highly branched macromolecules with well-defined, monodispersed, nanoscale structures. The coordination structure was confirmed by NMR, XPS, and MALDI-TOF spectra. The coordination increased the uptake of both selenium and platinum in HepG2 cells as indicated by ICP-MS, probably because the coordination of supra-amphiphile increased the solubility of cisplatin and changed the uptake mechanism of the compound. Dendrimers of different generations (DLG1Se, DLG2Se, DLG3Se) showed different uptake behaviors, with generation 1 DLG1Se having the highest uptake. They all showed high anticancer activity in Balb/c mice implanted with $4 \mathrm{~T} 1$ breast cancer cells. A similar strategy could be used to fabricate a drug delivery hydrogel transited from thermal responsive micelles,$^{50}$ a layer-by-layer thin film when heavier chalcogens ${ }^{51}$ were employed, indicating that the $\mathrm{Se}-\mathrm{M}^{n+}$ supra-amphiphile approach is generic.

We believe that the novel selenium-containing supraamphiphiles are exciting materials for nanomedicine. ${ }^{52}$ First, selenium species are closely related to reactive oxygen species in the human body. We believe regulating the ROS level in living systems using selenium compounds is an appealing research area that could greatly impact the nanomedicine field. Secondly, considering the light responsiveness and tunable bond dissociation energies of different $\mathrm{Se} \cdots \mathrm{X}$ bonds, the seleniumcontaining supra-amphiphiles could work as a photo uncaging strategy to selectively deliver drug molecules, proteins, cytokine or nucleic acids to targeted sites. In addition, we are also developing some selenium-containing supra-amphiphiles for cancer immunotherapy by utilizing the immune activity of selenium species.

\section{Conclusions}

In summary, we have reviewed the recent advances in seleniumcontaining supra-amphiphiles. Conventional non-covalent interactions or Se $\cdots \mathrm{X}$ interactions could be used as the driving force for the supra-amphiphile formation. With the continuous efforts of scientists, $\mathrm{X}$ could so far include different elements, including Se itself, nitrogen and metal cations. These efforts empower chemists to have an expansive view of seleniumcontaining materials, in which the sensitive response and biofunctions could be incorporated into numerous systems by different supramolecular interactions without too much tedious chemical synthesis. However, currently there are still unsolved problems. For the diselenide exchange reaction, most of our understanding is still qualitative. Measuring the Gibbs free energy change, entropy and enthalpy change, activation energy and quantum yield of the photoreaction could not only provide more physical organic insights into the nature of this important reaction, but also guide us along applying the diselenide chemistry to numerous fields, like external-stimulitriggered targeted delivery, remotely-controlled self-healing materials, and light responsive soft robots. Additionally, mechanisms for some of our exciting biological discoveries are not fully explored. The uptake mechanism of EG-Se/Pt is still not clear. And the reason behind the ROS regulating ability 
is also worth further investigations. The questions of how far we can push forward the system need more collaboration with physicians, biologists and support from industry. We believe that a broader impact can only be built if people from different backgrounds truly appreciate the science we are developing and apply it to their own research.

Apart from the example described above, other Se-X bonds could also be used for supra-amphiphiles. As a heavier chalcogen, selenium has a stronger binding with gold. ${ }^{53}$ By choosing the chalcogen species, we can fabricate different compounds with tuneable bond dissociation energies, and further develop programmable functional supra-amphiphilic systems. Se-O, $\mathrm{Se}-\mathrm{S},{ }^{35} \mathrm{Se}-\mathrm{Te},{ }^{37}$ and selenoester bonds could be potential dynamic covalent linkers to connect various moieties together. Although this review mostly focused on static self-assembly, the responsiveness of selenium-containing supra-amphiphiles may potentially open a new way for dynamic self-assembly, in which continuous dissipation of energy are essential. The supramolecular approach could be of interest in many applications at the interfaces of chemical biology, materials science, and nanomedicine.

\section{Conflicts of interest}

There are no conflicts to declare.

\section{Acknowledgements}

The authors would like to thank the National Science Foundation for Distinguished Young Scholars (Grant 21425416), the National Basic Research Plan of China (Grant 2018YFA0208900), and the Foundation for Innovative Research Groups of the National Natural Science Foundation of China (Grant 21821001).

\section{Notes and references}

1 J. N. Israelachvili, D. J. Mitchell and B. W. Ninham, J. Chem. Soc., Faraday Trans. 2, 1976, 72, 1525-1568.

2 Y. Chang, Y. Jiao, H. E. Symons, J. F. Xu, C. F. J. Faul and X. Zhang, Chem. Soc. Rev., 2019, 48, 989-1003.

3 X. Zhang and C. Wang, Chem. Soc. Rev., 2011, 40, 94-101.

4 C. Wang, Z. Wang and X. Zhang, Acc. Chem. Res., 2012, 45, 608-618.

5 H. Ringsdorf, B. Schlarb and J. Venzmer, Angew. Chem., Int. Ed. Engl., 1988, 27, 113-158.

6 G. M. Whitesides and B. Grzybowski, Science, 2002, 295, 2418-2421.

7 C. Wang, Z. Wang and X. Zhang, Small, 2011, 7, 1379-1383.

8 Y. Wang, H. Xu and X. Zhang, Adv. Mater., 2009, 21, 2849-2864.

9 K. Liu, Y. Kang, Z. Wang and X. Zhang, Adv. Mater., 2013, 25, 5530-5548.

10 H. Xu, W. Cao and X. Zhang, Acc. Chem. Res., 2013, 46, 1647-1658.
11 W. Cao, L. Wang and H. Xu, Nano Today, 2015, 10, 717-736.

12 N. Ma, Y. Li, H. S. Xu, Z. Wang and X. Zhang, J. Am. Chem. Soc., 2010, 132, 442-443.

13 N. Ma, Y. Li, H. Ren, H. Xu, Z. Li and X. Zhang, Polym. Chem., 2010, 1, 1609-1614.

14 L. Wang, W. Cao, Y. Yi and H. Xu, Langmuir, 2014, 30, 5628-5636.

15 J. Liu, Y. Pang, J. Chen, P. Huang, W. Huang, X. Zhu and D. Yan, Biomaterials, 2012, 33, 7765-7774.

16 H. Xu, J. Gao, Y. Wang, Z. Wang, M. Smet, W. Dehaen and X. Zhang, Chem. Commun., 2006, 796-798.

17 X. Zhang, H. Xu, Z. Dong, Y. Wang, J. Liu and J. Shen, J. Am. Chem. Soc., 2004, 126, 10556-10557.

18 S. Ji, W. Cao, Y. Yu and H. Xu, Adv. Mater., 2015, 27, 7740-7745.

19 L. Wang, K. Zhu, W. Cao, C. Sun, C. Lu and H. Xu, Polym. Chem., 2019, 10, 2039-2046.

20 L. Yu, M. Zhang, F.-S. Du and Z.-C. Li, Polym. Chem., 2018, 9, 3762-3773.

21 L. Pauling, The nature of the chemical bond and the structure of molecules and crystals: an introduction to modern structural chemistry, Cornell University Press, Ithaca, N.Y., 1960.

22 N. K. Kildahl, J. Chem. Educ., 1995, 72, 423-424.

23 R. E. Huber and R. S. Criddle, Arch. Biochem. Biophys., 1967, 122, 164-173.

24 D. Steinmann, T. Nauser and W. H. Koppenol, J. Org. Chem., 2010, 75, 6696-6699.

25 D. Van Vranken and G. Weiss, Introduction to bioorganic chemistry and chemical biology, Garland Science, Taylor \& Francis Group, New York, 2013.

26 J. T. Rotruck, A. L. Pope, H. E. Ganther, A. B. Swanson, D. G. Hafeman and W. G. Hoekstra, Science, 1973, 179, 588-590.

27 R. J. Hondal, B. L. Nilsson and R. T. Raines, J. Am. Chem. Soc., 2001, 123, 5140-5141.

28 P. Han, N. Ma, H. Ren, H. Xu, Z. Li, Z. Wang and X. Zhang, Langmuir, 2010, 26, 14414-14418.

29 W. Cao, X. Zhang, X. Miao, Z. Yang and H. Xu, Angew. Chem., Int. Ed., 2013, 52, 6233-6237.

30 W. Cao, Y. Gu, T. Li and H. Xu, Chem. Commun., 2015, 51, 7069-7071.

31 F. Fan, S. Gao, S. Ji, Y. Fu, P. Zhang and H. Xu, Mater. Chem. Front., 2018, 2, 2109-2115.

32 S. Ji, W. Cao, Y. Yu and H. Xu, Angew. Chem., Int. Ed., 2014, 53, 6781-6785.

33 S. Ji, J. Xia and H. Xu, ACS Macro Lett., 2016, 5, 78-82.

34 J. Xia, T. Li, C. Lu and H. Xu, Macromolecules, 2018, 51, 7435-7455.

35 F. Fan, S. Ji, C. Sun, C. Liu, Y. Yu, Y. Fu and H. Xu, Angew. Chem., Int. Ed., 2018, 57, 16426-16430.

36 J. Xia, P. Zhao, S. Pan and H. Xu, ACS Macro Lett., 2019, 8, 629-633.

37 C. Liu, J. Xia, S. Ji, Z. Fan and H. Xu, Chem. Commun., 2019, 55, 2813-2816.

38 J. Xia, S. Ji and H. Xu, Polym. Chem., 2016, 7, 6708-6713. 
39 J. Xia, P. Zhao, K. Zheng, C. Lu, S. Yin and H. Xu, Angew. Chem., Int. Ed., 2019, 58, 542-546.

40 A. J. Mukherjee, S. S. Zade, H. B. Singh and R. B. Sunoj, Chem. Rev., 2010, 110, 4357-4416.

41 G. Mugesh and H. B. Singh, Chem. Soc. Rev., 2000, 29, 347-357.

42 Y. Yi, S. Fa, W. Cao, L. Zeng, M. Wang, H. Xu and X. Zhang, Chem. Commun., 2012, 48, 7495-7497.

43 Y. Yi, H. Xu, L. Wang, W. Cao and X. Zhang, Chem. - Eur. J., 2013, 19, 9506-9510.

44 X. Huang, R. Fang, D. Wang, J. Wang, H. Xu, Y. Wang and X. Zhang, Small, 2015, 11, 1537-1541.

45 W. Cao, Y. Li, Y. Yi, S. Ji, L. Zeng, Z. Sun and H. Xu, Chem. Sci., 2012, 3, 3403-3408.

46 W. Cao, Y. Gu, M. Meineck, T. Li and H. Xu, J. Am. Chem. Soc., 2014, 136, 5132-5137.

47 L. Zeng, Y. Li, T. Li, W. Cao, Y. Yi, W. Geng, Z. Sun and H. Xu, Chem. - Asian J., 2014, 9, 2295-2302.
48 F. Li, T. Li, X. Han, H. Zhuang, G. Nie and H. Xu, ACS Biomater. Sci. Eng., 2017, 4, 1954-1962.

49 T. Li, M. Smet, W. Dehaen and H. Xu, ACS Appl. Mater. Interfaces, 2016, 8, 3609-3614.

50 J. Luan, W. Shen, C. Chen, K. Lei, L. Yu and J. Ding, RSC Adv., 2015, 5, 97975-97981.

51 W. Cao, L. Wang and H. Xu, Chem. Commun., 2015, 51, 5520-5522.

52 H. Chen, Z. Gu, H. An, C. Chen, J. Chen, R. Cui, S. Chen, W. Chen, X. Chen, X. Chen, Z. Chen, B. Ding, Q. Dong, Q. Fan, T. Fu, D. Hou, Q. Jiang, H. Ke, X. Jiang, G. Liu, S. Li, T. Li, Z. Liu, G. Nie, M. Ovais, D. Pang, N. Qiu, Y. Shen, H. Tian, C. Wang, H. Wang, Z. Wang, H. Xu, J.-F. Xu, X. Yang, S. Zhu, X. Zheng, X. Zhang, Y. Zhao, W. Tan, X. Zhang and Y. Zhao, Sci. China: Chem., 2018, 61, 1503-1552.

53 W. Xiang, Z. Li, C. Q. Xu, J. Li, W. Zhang and H. Xu, Chem. Asian J., 2019, 14, 1481-1486. 